\title{
Anesthesiologic effects of transperitoneal versus extra- peritoneal approach during robot-assisted radical prostatectomy: results of a prospective randomized study
}

Fabrizio Dal Moro ${ }^{1}$, Alessandro Crestani ${ }^{1}$, Claudio Valotto ${ }^{1}$, Andrea Guttilla ${ }^{1}$, Rodolfo Soncin ${ }^{1}$, Angelo Mangano ${ }^{2}$, Filiberto Zattoni ${ }^{1}$

${ }^{1}$ Dipartimento di Scienze Chirurgiche, Oncologiche e Gastroenterologiche, Università degli Studi di Padova, Italia; ${ }^{2}$ Dipartimento di Anestesiologia, Azienda Ospedaliera di Padova, Padova, Italia

\section{ABSTRACT}

Objectives: To compare the effects of $\mathrm{CO}_{2}$ insufflation on hemodynamics and oxygen levels and on acid-base level during Robot-Assisted Radical Prostatectomy (RARP) with transperitoneal (TP) versus extra-peritoneal (EP) accesses.

Materials and Methods: Sixty-two patients were randomly assigned to TP (32) and EP (30) to RARP. Pre-operation data were collected for all patients. Hemodynamic, respiratory and blood acid-base parameters were measured at the moment of induction of anesthesia (T0), after starting $\mathrm{CO}_{2}$ insufflation (T1), and at 60 (T2) and 120 minutes (T3) after insufflation. In all cases, the abdominal pressure was set at $15 \mathrm{mmHg}$. Complications were reported according to the Clavien-Dindo classification. Student's two-t-test, with a significance level set at $\mathrm{p}<0.05$, was used to compare categorical values between groups. The Mann-Whitney U-test was used to compare the median values of two nonparametric continuous variables.

Results: The demographic characteristics of the patients in both groups were statistically comparable. Analysis of intra-operative anesthesiologic parameters showed that partial $\mathrm{CO}_{2}$ pressure during EP was significantly higher than during TP, with a consequent decrease in arterial $\mathrm{pH}$. Other parameters analysed were similar in the two groups. Postoperative complications were comparable between groups. The most important limitations of this study were the small size of the patient groups and the impossibility of maintaining standard abdominal pressure throughout the operational phases, despite attempts to regulate it.

Conclusions: This prospective randomized study demonstrates that, from the anesthesiologic viewpoint, during RARP the TP approach is preferable to EP, because of lower $\mathrm{CO}_{2}$ reabsorption and risk of acidosis.

\section{ARTICLE INFO}

\section{Key words:}

Prostatectomy; Robotic Surgical Procedures; Laparoscopy;

Anesthesiology

Int Braz J Urol. 2015; 41: 466-72

Submitted for publication:

April 21, 2014

Accepted after revision:

September 30, 2014

\section{INTRODUCTION}

Robot-Assisted Radical Prostatectomy (RARP) can be performed via either transperitoneal (TP) or total extra-peritoneal (EP) approaches. There are some contraindications for TP endos- copic surgery, such as obesity or intraperitoneal adhesions, which may make the EP approach more attractive. EP is also believed to follow open surgery principles more closely, because the peritoneal cavity is not violated and the peritoneum functions as a natural bowel retractor, thereby 
preventing bowel displacement into the surgical field. Another advantage of the EP approach is the possibility of decreasing the risk of intra-abdominal complications such as bowel injury.

Nevertheless, TP is the most frequently used approach to RARP, perhaps because a larger working space is created than during EP.

During laparo-endoscopy, carbon dioxide $\left(\mathrm{CO}_{2}\right)$ insufflation into both peritoneal and extra-peritoneal cavities produces a wide range of pathophysiological hemodynamic changes. Vigilance in monitoring and diligence in management are essential in order to prevent complications.

Although several studies comparing extra/ retroperitoneal and TP approaches for laparoscopic or robotic surgical interventions (1) have focused on functional and oncological outcomes, the literature contains few reports on the anesthesiologic effects of the two approaches in animal models and only one concerning RARP in humans (2).

This prospective randomized study assesses the effects of EP and TP $\mathrm{CO}_{2}$ insufflation on hemodynamic parameters and oxygen transport in a group of patients undergoing RARP for prostate cancer.

\section{MATERIALS AND METHODS}

In this study, 62 patients were randomly assigned to TP (32) or EP (30) RARP. Pre-operation data, including age, BMI, American Society of Anesthesiologists class, PSA levels, prostate volume, clinical stage and Gleason scores were collected prospectively Table- 1 .

In order to reduce any confounding factor due to heterogeneity among surgeons, this study was designed so that, in all cases, two similarly experienced surgeons performed the initial approach (FDM and CV), and another experienced surgeon (FZ) performed the actual prostatectomy, using a standardized method.

The time required for each operative step was recorded. The following hemodynamic, respiratory and oxygen transport parameters were measured: $\mathrm{pH}$, partial arterial pressure of oxygen $\left(\mathrm{PaO}_{2}\right)$, partial arterial pressure of $\mathrm{CO}_{2}\left(\mathrm{PaCO}_{2}\right)$, tidal volume, respiratory frequency, ventilation pressure, and volume/minute, recorded for each patient at the moment of induction of anesthesia (T0), after starting $\mathrm{CO}_{2}$ insufflation (T1), and at 60 (T2) and 120 minutes (T3) after insufflation.

Complications were defined and graded according to the Clavien-Dindo classification (3).

Exclusion criteria included prior abdominal surgery. In this series there were no patients with chronic pulmonary disease included since they ideally were not candidate for robotic prostatectomy.

We received approval of the ethical committee of the institution and all patients signed a consent form.

\section{ANESTHESIA AND SURGICAL TECHNIQUE}

\section{Anesthesia}

Patients' respiratory rate was set at 10 breaths per minute. Tidal volume was set at 8-10 $\mathrm{mL} / \mathrm{kg}$ and adjusted by means of repeated arterial blood gas analyses to maintain $\mathrm{PaO}_{2}$ and $\mathrm{pH}$ within the normal ranges of $32-45 \mathrm{mmHg}$ and $7.34-$ 7.47 , respectively.

To evaluate hemodynamics and gas exchange during $\mathrm{CO}_{2}$ insufflation, first arterial blood gas analyses were performed at the moment of induction of anesthesia as a baseline value.

Measurements were taken for heart rate, mean arterial blood pressure, and end-tidal $\mathrm{CO}_{2}$. Arterial (radial) and central venous (internal jugular) blood samples were taken for gas analyses of $\mathrm{PaO}_{2}, \mathrm{PaCO}_{2}$ pressure, and mixed venous oxygen saturation.

\section{Surgical technique}

\section{EP approach}

Under general anesthesia, the patient was placed in the Trendelenburg position $\left(20^{\circ}\right)$ with legs apart. A 20-Fr Foley catheter was inserted into the bladder. The skin was incised transversely just below the umbilicus. The anterior rectus sheet was incised vertically over $1 \mathrm{~cm}$. The pre-peritoneal space was then freed by finger dissection and further developed with a balloon trocar (Tyco, Norwalk, CN), inflated by 20-24 pumping actions until the extra-peritoneal space was appropriately created. The first $8-\mathrm{mm}$ robot trocar (Intui- 
Table 1 - Demographic and clinical characteristics of extra-peritoneal and transperitoneal robot-assisted radical prostatectomy.

\begin{tabular}{|c|c|c|c|}
\hline & EP Approach & TP Approach & $p$ value \\
\hline \multicolumn{4}{|l|}{ Age } \\
\hline Mean (SD) & $61.3( \pm 6.57)$ & $64.09( \pm 6.56)$ & 0.103 \\
\hline \multicolumn{4}{|l|}{ ASA class (\%) } \\
\hline$|-| \mid$ & $100 \%$ & $100 \%$ & 1 \\
\hline III-IV & 0 & 0 & \\
\hline \multicolumn{4}{|l|}{ BMI $\left(\mathrm{kg} / \mathrm{m}^{2}\right)$} \\
\hline Mean (SD) & $25.91( \pm 2.56)$ & $26.9( \pm 3.23)$ & 0.588 \\
\hline \multicolumn{4}{|c|}{ Pre-operative PSA (ng/mL) } \\
\hline Mean (SD) & $6.71( \pm 3.99)$ & $7.59( \pm 4.04)$ & 0.469 \\
\hline \multicolumn{4}{|c|}{ Prostate volume (cc) } \\
\hline Mean (SD) & $39.16( \pm 16.95)$ & $43.52( \pm 13.33)$ & 0.427 \\
\hline \multicolumn{4}{|c|}{ Bioptical GI. Score, n (\%) } \\
\hline$\leq 6$ & $19(63 \%)$ & $18(56 \%)$ & \\
\hline 7 & $9(30 \%)$ & $10(31 \%)$ & 0.238 \\
\hline $8-10$ & $2(7 \%)$ & $4(13 \%)$ & \\
\hline \multicolumn{4}{|c|}{ Clinical stage, n (\%) } \\
\hline T1c & $21(70 \%)$ & $27(84.37 \%)$ & \\
\hline T2a & $3(10 \%)$ & $2(6.25 \%)$ & 0.220 \\
\hline $\mathrm{T} 2 \mathrm{~b}$ & $4(13.3 \%)$ & $3(9.37 \%)$ & \\
\hline T2c & $1(3.33 \%)$ & 0 & \\
\hline
\end{tabular}

$\mathbf{S D}=$ standard deviation; $\mathbf{A S A}=$ American Society of Anesthesiologists

tive Surgical, Sunnyvale, CA) on the left side was bluntly introduced about four fingers laterally to the sub-umbilical incision, on the line joining the incision and the left anterior iliac crest. The 12mm optic trocar was then introduced and insufflation was started (intra-abdominal pressure 15 $\mathrm{mmHg}$ ). Inspection of the extra-peritoneal space was performed with the $0^{\circ} 3 \mathrm{D}$ da Vinci ${ }^{\circledR}$ camera. Under direct vision, the camera itself can be used to increase the size of the extra-peritoneal space by being made to sweep the peritoneal borders gently latero-cranially. The second $8-\mathrm{mm}$ robot trocar can be placed under visual control in order to reflect the previously placed one. The 12$\mathrm{mm}$ assistant trocar on the right side was placed cranio-medially to the right anterior iliac crest. Another 8-mm robot trocar was placed under vision on the left side, cranio-medially to the left anterior iliac crest for the fourth arm of the robot.
The 5-mm assistant trocar for the suction device was placed in between, about $2 \mathrm{~cm}$ cranially to the right robotic and the optic trocar. The abdominal wall was slightly lifted by the camera-arm trocar ("laparo-lift"). Lastly, the robot was docked.

TP approach

Under general anesthesia, the patient was placed in the Trendelenburg position (33) with legs apart. A 20-Fr Foley catheter was inserted into the bladder. The skin was incised vertically just above the umbilicus. The anterior rectus sheet was incised vertically over $1 \mathrm{~cm}$. The $12-\mathrm{mm}$ optic trocar was then introduced and a pneumoperitoneum was created (intra-abdominal pressure 15 $\mathrm{mmHg}$ ). Inspection of the abdominal cavity was performed with the $0^{\circ} 3 \mathrm{D}$ da Vinci ${ }^{\circledR}$ camera. Then the first 8-mm robot trocar on the right side was placed under vision, about four fingers laterally 
to the supra-umbilical incision on the line joining the sub-umbilical incision and the right anterior iliac crest. Another 8-mm robot trocar was placed specularly to the first one on the left side. A third 8-mm robot trocar for the fourth robotic arm was placed 2-4 inches cranially to the left anterior iliac crest. The distance of this trocar to the iliac crest depends on the morphology of the patient. A 12$\mathrm{mm}$ assistant trocar for the suction device and Hem-o-lok ${ }^{\circledR}$ positioning was placed in between, about $2 \mathrm{~cm}$ cranially to the right robotic and the optic trocar. Lastly, the robot was docked.

\section{Prostatectomy}

In all cases, standard pelvic lymphadenectomy was performed before prostatectomy. After removal of the prostate and before anastomosis, the dorsal vein complex was suture-ligated: during this phase, intra-cavity pressure was increased to $20 \mathrm{mmHg}$.

\section{Statistical analysis}

Student's two-tailed $t$-test, with a significance level set at $\mathrm{p}<0.05$, was used to compare the categorical values between groups. The MannWhitney U-test was used to compare the median values of two nonparametric continuous variables. Statistical significance was $\mathrm{p}<0.05$.

\section{RESULTS}

The demographic characteristics of patients in the two groups were statistically comparable Table- 1 .

In the EP group, no patient was converted to the TP approach because of an unapproachable Retzius space or technical difficulties. In two patients, small lacerations in the peritoneum were noted, and procedures were completed extra-peritoneally.

Complications, according to the Dindo-Clavien Classification, are listed in Table-2, showing not statistically significant differences between the two groups. No open conversions were needed.

Analysis of the times required for each step of RARP only revealed a difference in the time for trocar positioning, which was significantly higher in $\mathrm{EP}$ (mean $31 \mathrm{~min} ; \mathrm{SD}_{ \pm} 11$ ) than $\mathrm{TP}$ (mean 10 $\left.\min ; \mathrm{SD}_{ \pm 2}\right), \mathrm{p}<0.001$.

Mean time to perform standard pelvic lymphadenectomy was not statistically different $(p=0.316)$ between two groups. In EP group was 23 min $\left(\mathrm{SD}_{ \pm} 8\right)$ and in $\mathrm{TP}$ group was $21 \mathrm{~min}\left(\mathrm{SD}_{ \pm} 6\right)$.

Mean total operative time was $146 \mathrm{~min}$ for EP and 128 min for TP approach, $\mathrm{p}=0.19$.

Mean estimated blood loss was $220 \mathrm{~mL}$ for $\mathrm{EP}$ and $312 \mathrm{~mL}$ for TP approach, $\mathrm{p}=0.289$.

Table 2 - Post operative complications according to Clavien-Dindo classification.

\begin{tabular}{|c|c|c|c|}
\hline & EP Approach (30) & TP Approach (32) & $p$ value \\
\hline All Grades & Overall: $20 \%$ & Overall: $18.75 \%$ & ns \\
\hline \multirow[t]{2}{*}{ Grade I-II } & Overall: $16.7 \%$ & Overall: 18.75\% & \\
\hline & $\begin{array}{l}\text { Urinary infections w/o therapy (10\%); Blood } \\
\text { Transfusion (3.3\%); Urinary Leakage }(3.3 \%)\end{array}$ & $\begin{array}{c}\text { Not infectious diarrhea }(6.25 \%) \text {; Urinary } \\
\text { infections w/o therapy }(6.25 \%) \text {; Blood } \\
\text { Transfusion }(6.25 \%)\end{array}$ & ns \\
\hline \multirow[t]{2}{*}{ Grade III } & Overall: 3.3\% & & \\
\hline & $\begin{array}{l}\text { Lymphocele requiring percutaeous drainage } \\
\qquad(3.3 \%)\end{array}$ & - & ns \\
\hline Grade IV & - & - & - \\
\hline Grade V & - & - & - \\
\hline
\end{tabular}


The heart rate did not change significantly during either of the surgical approaches.

Although mean arterial blood pressure and cardiac output did increase significantly during TP insufflation, although not during EP insufflation, the differences between the methods were not statistically significant.

Gas exchange parameters are listed in Table-3.

Figure-1 shows changes in $\mathrm{PaCO}_{2}$ (Panel A) and blood artery $\mathrm{pH}$ (Panel B) during RARP with the EP and TP approaches.

Analysis of intra-operative anesthesiologic parameters showed that $\mathrm{PaCO}_{2}$ was significantly higher during EP than during TP at times T2 and T3 $(p=0.0125)$, with a consequent significant decrease in arterial $\mathrm{pH}(\mathrm{p}=0.0268)$. Other parameters analysed were similar in both groups.

\section{DISCUSSION}

In our study analysis of intra-operative anesthesiologic parameters showed that partial $\mathrm{CO}_{2}$ pressure during EP was significantly higher than during TP, with a consequent decrease in arterial $\mathrm{pH}$. Furthermore tidal volume was higher in EP group although there was not a statistically significant difference.

$\mathrm{CO}_{2}$ absorption during laparo-endoscopic surgery is a challenging anesthesiologic problem, and may lead to hypercarbia and acidosis. Clearance of $\mathrm{CO}_{2}$ is related to adequate alveolar ventilation, and a rapid increase in $\mathrm{CO}_{2}$ levels may be compensated by hyperventilation of the lungs.

Only a small proportion of $\mathrm{CO}_{2}$ is dissolved in blood and delivered directly to the lungs.

Most of it combines with water in red blood cells to form carbonic acid, which then dissociates into hydrogen and bicarbonate, according to the formula:

$$
\mathrm{CO}_{2}+\mathrm{H}_{2} \mathrm{O} \leftrightarrow \mathrm{H}_{2} \mathrm{CO}_{3} \leftrightarrow \mathrm{H}^{+}+\mathrm{HCO}_{3}
$$

$\mathrm{CO}_{2}$ absorbed through the peritoneum is handled in the same manner and, ultimately, is eliminated by respiratory exchange in the lungs. While the patient is under general anesthesia, minute ventilation volumes must be increased to maintain normocarbia.

Although the increase in $\mathrm{PaCO}_{2}$ is not fully compensated by hyperventilation, most healthy patients can easily adapt to the increase in end-tidal $\mathrm{CO}_{2}$. However, some cannot tolerate the increased $\mathrm{CO}_{2}$ load during insufflation, and this condition may lead to myocardial depression and vasodilation. Conversely, severe acidemia and hypercapnia may depress heart performance, secondary to decreases in myocardial inotropy (4).

The patient counteracts these effects by centrally mediated sympathetic stimulation, which

Table 3 - Gas exchange parameters.

\begin{tabular}{lccccccccc}
\hline & \multicolumn{3}{c}{ Extra-Peritoneal approach } & \multicolumn{3}{c}{ Transperitoneal approach } & p value \\
\hline Parameters & T0 & T1 & T2 & T3 & T0 & T1 & T2 & T3 & \\
\hline pH & 7.464 & 7.427 & 7.376 & 7.374 & 7.46 & 7.448 & 7.417 & 7.413 & 0.0268 \\
PaO $_{2}$ ( $\left.\mathbf{m m H g}\right)$ & 157 & 159.2 & 153 & 167.3 & 141.2 & 143.6 & 152.3 & 154.1 & 0.5963 \\
PaCO $_{2}$ ( $\left.\mathbf{m m H g}\right)$ & 33.5 & 37.1 & 44.7 & 40.6 & 33.4 & 33.8 & 35.7 & 36.1 & 0.0125 \\
Tidal Vol. (mL) & 651 & 687 & 729 & 764 & 669 & 678 & 682 & 698 & 0.0693 \\
Resp. Freq. & 12 & 12 & 13 & 14 & 12 & 12 & 13 & 13 & 0.6459 \\
Press.Vent. (cmH $\mathbf{0})$ & 16 & 24 & 26 & 29 & 17 & 30 & 30 & 31 & 0.0986 \\
Pulmonary liters inflated/min & 7.7 & 8.1 & 9.6 & 12.6 & 7.8 & 8.6 & 9.2 & 9.5 & 0.4732 \\
\hline
\end{tabular}


Figure 1 - Arterial $\mathrm{CO}_{2}$ pressure $(\mathrm{A})$ and $\mathrm{pH}(\mathrm{B})$ during carbon dioxide $\left(\mathrm{CO}_{2}\right)$ insufflation in TP (red line) and EP (blue line) groups. Data are presented as means at moment of induction of anesthesia (TO), after starting $\mathrm{CO}_{2}$ insufflation (T1), and at 60 (T2) and 120 minutes (T3) after insufflation.
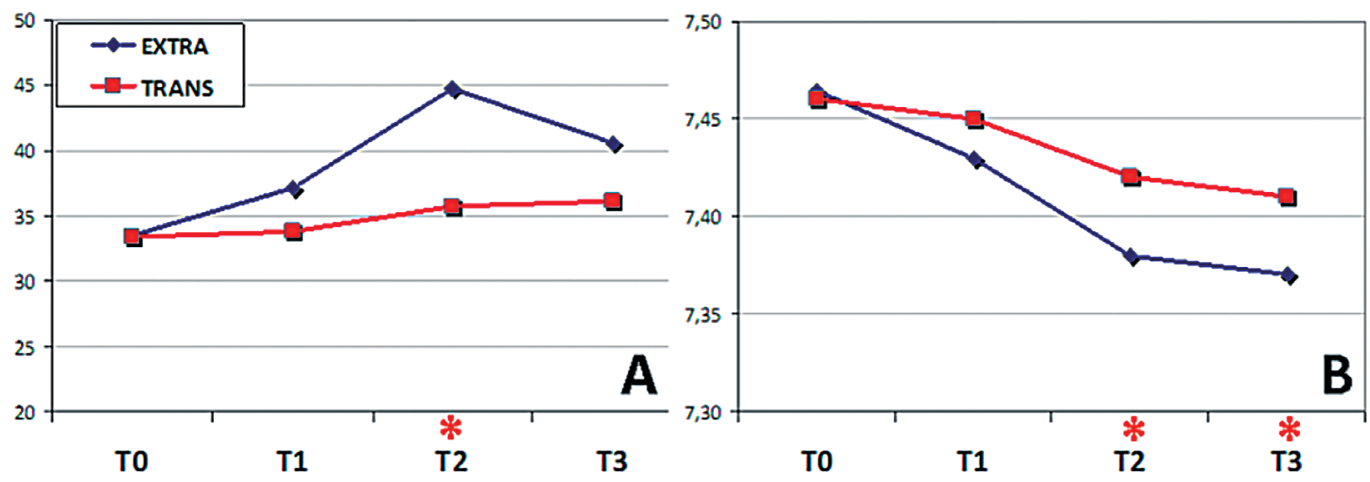

*TP vs EP groups, $p<0.05$.

causes persistent increases in blood pressure and heart rate, increasing catecholamine concentrations (5).

In addition, the pneumoperitoneum, because of direct compression of the diaphragm, leads to a significant reduction in forced expiratory volume, peak expiratory flow and forced vital capacity, with a consequent decrease in pulmonary compliance (6).

The extent of the hemodynamic effects of hypercarbia is also correlated to the patient's position: in the Trendelenburg position, abdominal contents are pushed against the diaphragm, reducing functional residual capacity and predisposing to atelectasis (5). In 1946, Case et al. (7) described a $15 \%$ decrease in lung volume in this position. The Trendelenburg position also increases central venous pressure, myocardial work, pulmonary venous pressure (decreasing pulmonary compliance), intra-cranial pressure (8) and intra-ocular pressure (9).

Our data concerning hemodynamic changes during RARP demonstrate that the EP approach is associated with hemodynamic alterations similar to those observed during TP access, but with more rapid increases in partial $\mathrm{CO}_{2}$ pressure compared with $\mathrm{TP}$, starting 1 hour after the beginning of $\mathrm{CO}_{2}$ insufflation.

Despite substantially comparable operation times, and despite a less extreme Trendelenburg position in the EP approach and theoretically higher permeation of the gas into the bloodstream from the peritoneal cavity during TP access, in our experience EP RARP causes a significantly higher absorption of $\mathrm{CO}_{2}$ than $\mathrm{TP}$, resulting in more rapid acidosis.
These findings confirm the results of the only reported study comparing $\mathrm{CO}_{2}$ homeostasis during TP and EP RARP in humans (2): the authors reported a more marked acidosis during $\mathrm{CO}_{2}$ insufflation in EP than during TP.

The mechanisms responsible for these changes appear to be multifactorial and have not been completely clarified.

One mechanism causing higher $\mathrm{EP} \mathrm{CO}_{2}$ reabsorption may be direct intra-vascular uptake of $\mathrm{CO}_{2}$, perhaps facilitated, as suggested by Glascock et al. (10), by the disruption of microvascular and lymphatic channels during the creation of an extra-peritoneal cavity.

Another less well-known element playing a role in $\mathrm{CO}_{2}$ absorption may be the use of a suction device during TP: repeated suction during the operation causes a cyclic decrease-increase in intracavity pressure, which is less pronounced in the peritoneal cavity because of the larger space, in which suction leads to a dramatic and rapid decrease in pressure. Repeated rapid variations in pressure may act as a sort of pump which insufflates $\mathrm{CO}_{2}$ into the microvessels of the extra-peritoneal cavity, causing gas reabsorption.

Examining the clinical outcomes of our patients, in cases of acidosis and hypercarbia, the ventilation parameters were intra-operatively modified to maintain values under $30 \mathrm{mmHg}$ and $\mathrm{pH}>7.3$, respectively, increasing tidal volume and respiratory frequency, and successfully correcting $\mathrm{pH}$ alterations without complications. 
All of our patients performed a pelvic lymphadenectomy (PLND) independently to the risk group. This step inevitably prolonged the duration of the procedure in both groups. However the extension of the lymphadenectomy has been the same in both groups. We performed PLND in all of our patients for a prospective study (on going in that period) in order to evaluate a new nomogram on the risk of nodes metastasis in prostate cancer patients. The purpose of the study was to investigate whether patients actually classified as low risk (according to D'Amico risk groups) had in fact a risk close to zero to have nodes metastasis and to evaluate the predictive power of a new nomogram independently to the core's number evaluated at the biopsy. Nowadays the guidelines do not suggest to perform PLND in all patients because of the very low risk of nodes metastasis in low risk patients. However the nomograms proposed do not evaluate the total number of cores at the biopsy but only the percentage of positive cores.

There are several criticisms of our study: one of the most important is the impossibility of maintaining standard intra-cavity pressure throughout the operational phases, despite attempts to regulate it.

In addition, the statistical power of the small sample size of the two groups may have been insufficient to detect certain differences between them.

Nevertheless, our findings bring to the attention-not only of urologists but also of anesthesiologists-the possible hemodynamic effects of which approach is used for RARP. Although the choice is often dictated by the surgeon's preferences, the above data may help to tailor a safer approach for each patient, preferring when possible TP access, when $\mathrm{CO}_{2}$ reabsorption could be more dangerous and difficult to manage intra-operatively by the anesthesiologist.

This prospective randomized study demonstrates that TP and EP RARP are comparable, considering the times of the single steps of the operation. From the anesthesiologic viewpoint, during RARP the TP approach is preferable, because there is less $\mathrm{CO}_{2}$ reabsorption and risk of acidosis. These data should consequently be interpreted with caution, until they can be replicated in other settings and in other types of studies.

\section{REFERENCES}

1. Ficarra V, Novara G, Artibani W, Cestari A, Galfano A, Graefen $M$, et al. Retropubic, laparoscopic, and robot-assisted radical prostatectomy: a systematic review and cumulative analysis of comparative studies. Eur Urol. 2009;55:1037-63.

2. Meininger $D$, Byhahn $C$, Wolfram $M$, Mierdl S, Kessler $P$, Westphal K. Prolonged intraperitoneal versus extraperitoneal insufflation of carbon dioxide in patients undergoing totally endoscopic robot-assisted radical prostatectomy. Surg Endosc. 2004;18:829-33.

3. Dindo D, Demartines N, Clavien PA. Classification of surgical complications: a new proposal with evaluation in a cohort of 6336 patients and results of a survey. Ann Surg. 2004;240:205-13.

4. Youssef MA, Al Mulhim A. Physiologic effects of pneumoperitoneum in adults with sickle cell disease undergoing laparoscopic cholecystectomy (a case control study). Surg Endosc. 2008;22:1513-8.

5. Kanwer DB, Kaman L, Nedounsejiane M, Medhi B, Verma GR, Bala I. Comparative study of low pressure versus standard pressure pneumoperitoneum in laparoscopic cholecystectomy-a randomised controlled trial. Trop Gastroenterol. 2009;30:1714.

6. Hasukić S, Mesić D, Dizdarević E, Keser D, Hadziselimović S, Bazardzanović M. Pulmonary function after laparoscopic and open cholecystectomy. Surg Endosc. 2002;16:163-5.

7. Case EH, Stiles JA. The effect of various surgical positions on vital capacity. Anesthesiology. 1946;7:29-31.

8. Park EY, Koo BN, Min KT, Nam SH. The effect of pneumoperitoneum in the steep Trendelenburg position on cerebral oxygenation. Acta Anaesthesiol Scand. 2009;53:895-9.

9. Awad H, Santilli S, Ohr M, Roth A, Yan W, Fernandez S, et al. The effects of steep trendelenburg positioning on intraocular pressure during robotic radical prostatectomy. Anesth Analg. 2009;109:473-8.

10. Glascock JM, Winfield HN, Lund GO, Donovan JF, Ping ST, Griffiths DL. Carbon dioxide homeostasis during transperitoneal or extraperitoneal laparoscopic pelvic lymphadenectomy: a realtime intraoperative comparison. J Endourol. 1996;10:319-23.

Correspondence address: Fabrizio Dal Moro, MD Assistant Professor Dipartimento di Scienze Chirurgiche, Oncologiche e Gastroenterologiche, Università degli Studi di Padova Via Giustiniani 2, Padova, 35128, italy Fax: +3 904 9821-8757

E-mail: fabrizio.dalmoro@unipd.it 